\title{
Neurotrophic Support from Peripheral Nerve Tissue for Human Adrenal Chromaffin Cells (Co-Grafting) in the Treatment of Parkinson's Disease
}

\author{
I. Madrazo ${ }^{1}$, C. Cuevas1, R.E. Franco-Bourland ${ }^{2}$, M. Aguilera ${ }^{1}$, F. Ostrosky-Solis ${ }^{3}$ and H. Castrejon ${ }^{1}$. \\ ${ }^{1} U$ Invest Neurol Neurocir, Ctro Med Nal Siglo XXI, Inst Mex Seg Soc; ${ }^{2}$ Depto Bioquim, Inst Nal Nutricion \\ SZ; ${ }^{3}$ Depto Psicofisiol, Fac Psiciol, Univ Nal Aut Mex, Mexico City, Mexico
}

The successful brain graftings in hemiparkinsonian non-human primates using adrenal medullary (AM)/peripheral nerve (PN) co-grafts suggest that in humans, AM brain grafting for the treatment of Parkinson's disease (PD) could be improved by co-grafting with $\mathrm{PN}$. The beneficial effects of AM/PN co-grafting in non-human primates have been attributed to the neuronotrophic support of the PN for the improved survival and neurogenization of the grafted AM chromaffin cells. The potential of such support on the functional effect of AM grafts in PD patients has recently been evaluated using a continuous nerve growth factor intraputaminal infusion. Here we report our initial experience with $\mathrm{AM} / \mathrm{PN}$ co-grafting in four patients with $P D$.

The four parkinsonian subjects were three males (JTN, ORF, JRD) and one female (DBB), 41-51 years old, with an evolution of PD of 5-13 years, characterized by bradykinesia (BK) and rigidity (R) (JTN, DBB, ORF), and by tremor $(\mathrm{T})$ and rigidity (R) (JRD). All patients had been on levodopa (560-1000 $\mathrm{mg}$ ) for 4-8 years, and continue to respond well to the medication. Their presurgery scores on the Unified Parkinsonism Rating Scale (UPRS), the Hoehn \& Yahr (H\&Y), Schwab \& England (S\&E), and Madrazo (M) scales were, in "ON"/"OFF": JTN, $78 / 97,3 / 4,70 \% / 50 \%$, and $3 / 2$; DBB, 35/73, 2.5/4, $90 \% / 60 \%$, and $4 / 2$; ORF, $47 / 87,2.5 / 4,80 \% / 50 \%$, and $4 / 2$; and JRD, 33/79, 2.5/4, 80\%/.60\%, and 4/2. Using open microsurgery, autologous AM fragments were implanted to the right caudate nucleus together with freshly dissected, minced intercostal nerve tissue. Eighteen to 31 months after surgery, DBB and ORF have reduced their levodopa doses (in $\mathrm{mg} /$ day) from 750 to 500 and 660 , respectively, with mild (DBB) or moderate (ORF) dyskinesia as a side effect. JTN has increased his dosage from 560 to $660 \mathrm{mg} /$ day. The patients' postsurgery PD scores (UPRS, H\&Y, S\&E, and M) were: JTN, 27/54, 2/2.5, $90 \% / 80 \%$, and $4.5 / 4 ; \mathrm{DBB}, 25 / 61,2.5 / 4$, $90 \% / 50 \%$, and $4 / 2$; ORF, 53/75, $2 / 2.5,70 \% / 60 \%$, and $4.5 / 4 ; \mathrm{RJD}, 29 / 66,2.5 / 3,100 \% / 60 \%$, and $4 / 3$. The amount of change of their total UPRS scores in "ON" and "OFF" is shown in Fig. 1, and compared with the mean level of change in UPRS scoring of a heterogeneous population (HET POP) of 18 AM-alone transplanted PD patients. Only the functional recovery in "OFF" of JTN was comparable to the mean level of recovery of the AM-alone transplanted PD patients, and in "ON" was even higher by 16 points. The recoveries in "ON" and "OFF" of the other 3 patients were similar to each other, but much lower than that of the $18 \mathrm{AM}$-alone grafted PD patients; in "ON", ORF showed some deterioration. Regardless of the amount of change in their UPRS scorings, all patients showed improvement in the quality of their daily living activities. 

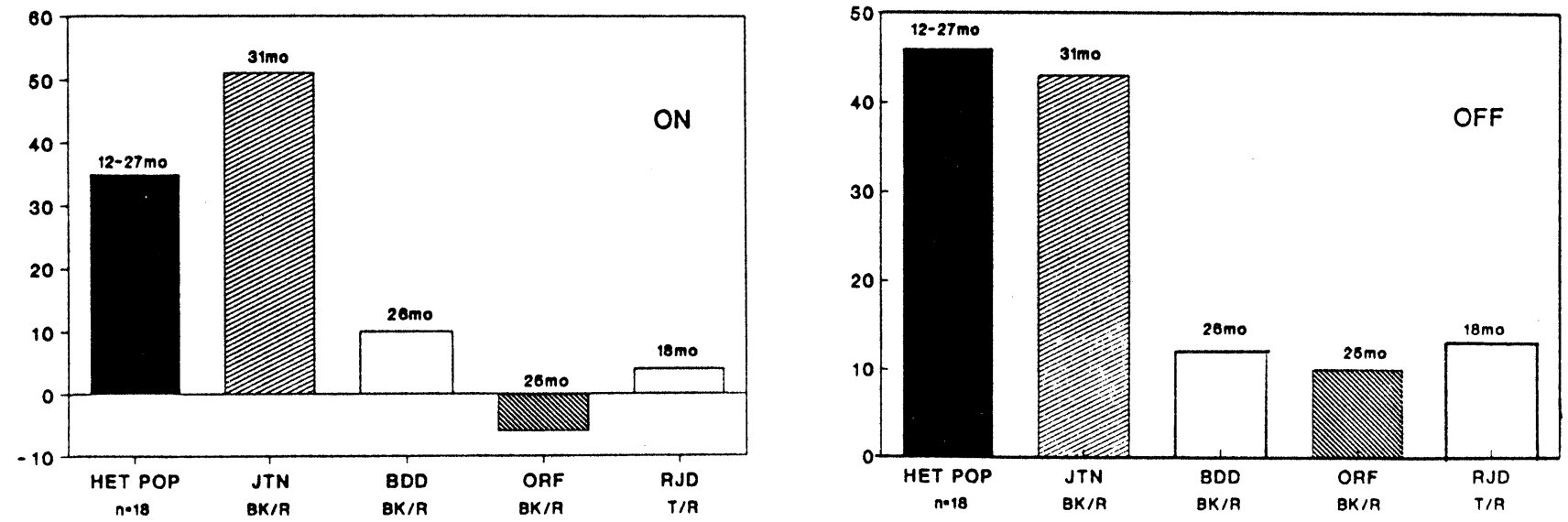

Fig. 1: AM/PN postsurgery evolution. The changes in total UPRS score are depicted for each patient (JTN, BDD, ORF, RJD) at the indicated follow-up times given in months (mo), and compared to the mean change in total UPRS score for a heterogeneous population (HET POP) of PD patients ( $n=18)$ 12-27 mo after autologous AM-alone brain grafting to the caudate nucleus. BK/R, bradykinesia/rigidity; $T / R$, tremor/rigidity. 

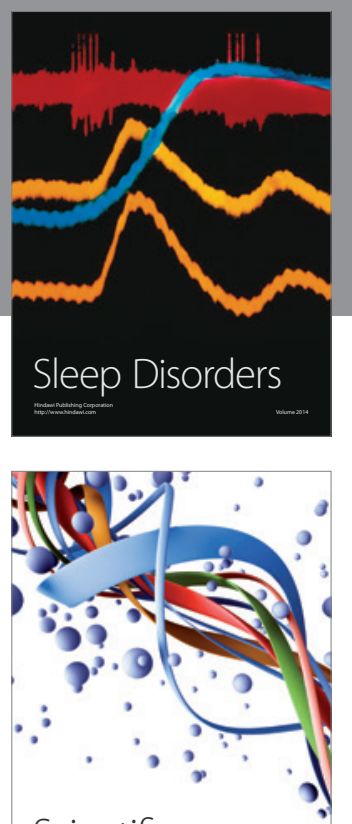

Scientifica
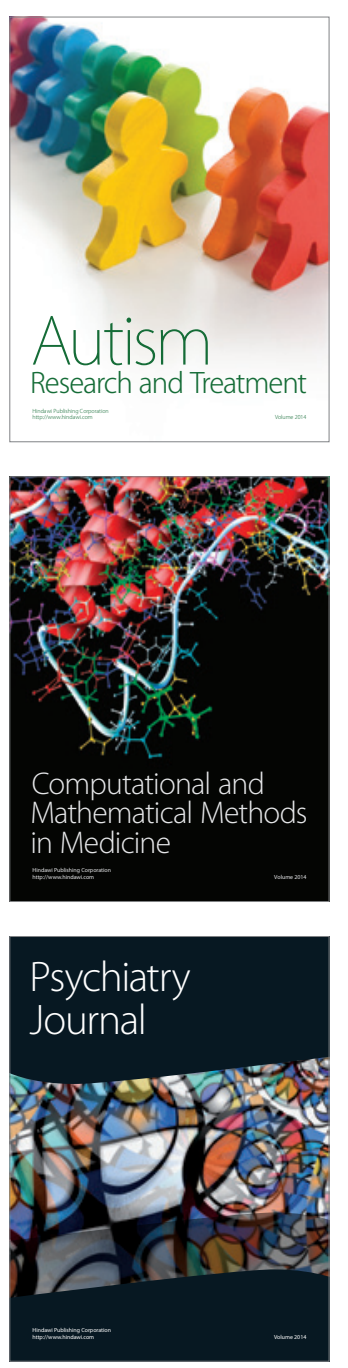
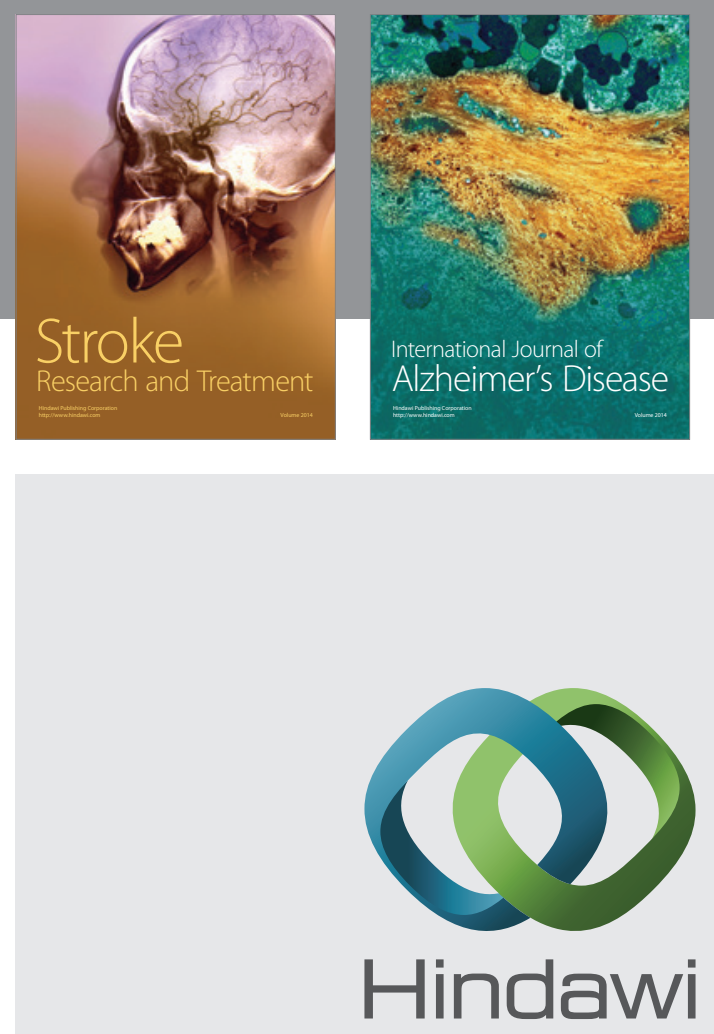

Submit your manuscripts at

http://www.hindawi.com
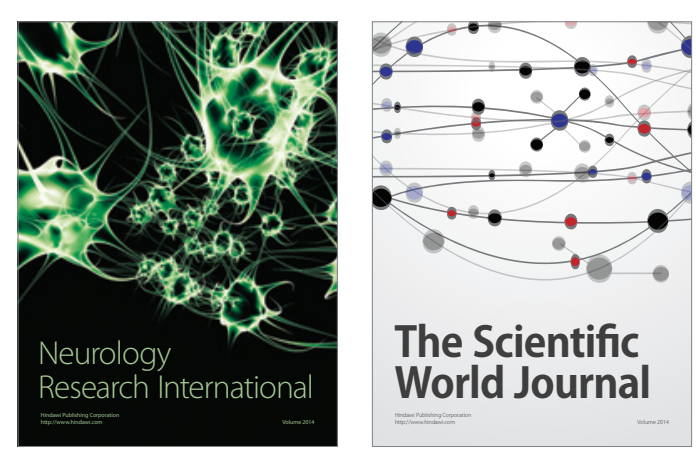

The Scientific World Journal

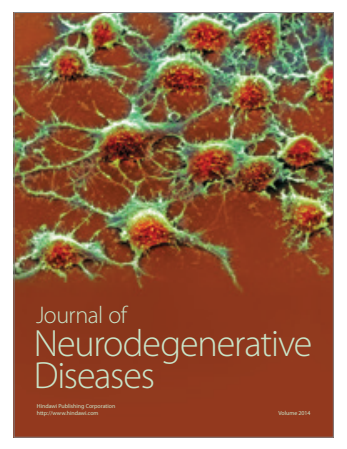

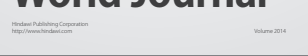

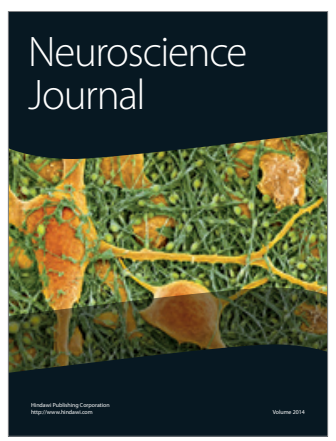

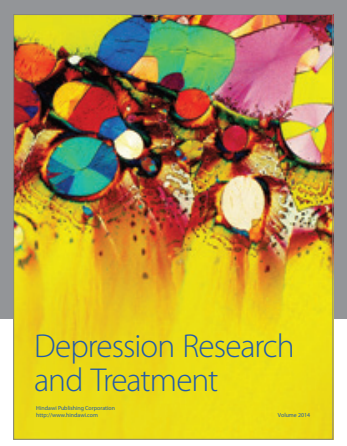
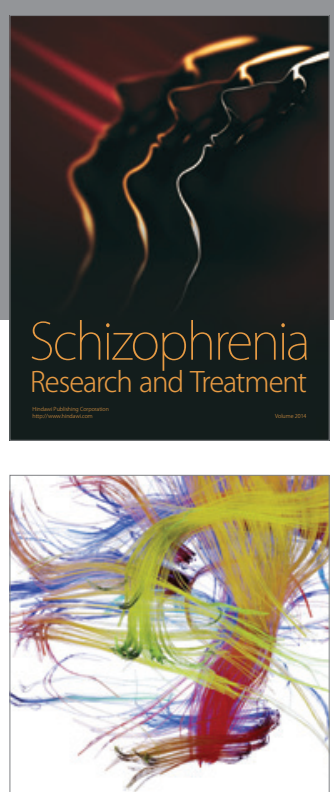

Brain Science

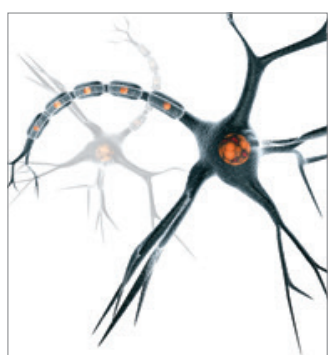

Neural Plasticity
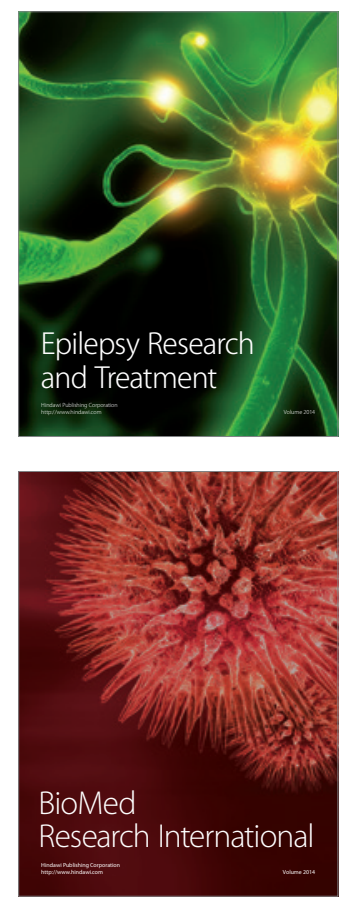

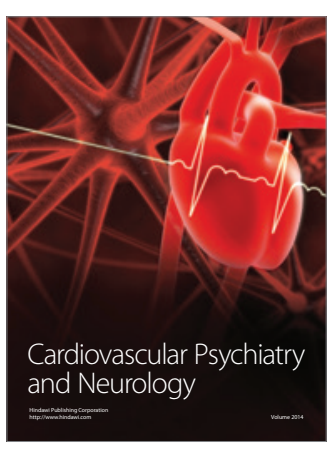

Parkinson's

Disease
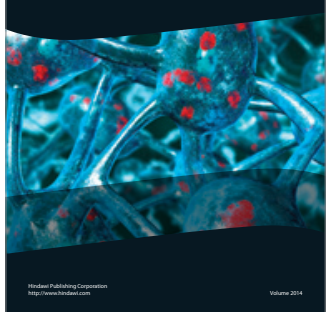